\title{
Type I planetary migration in a self-gravitating disk
}

\author{
Clément Baruteau ${ }^{1}$ and Frédéric Masset ${ }^{1,2}$ \\ ${ }^{1}$ Laboratoire AIM, CEA/DSM - CNRS - Université Paris Diderot \\ DAPNIA/Service d'Astrophysique, CEA/Saclay, 91191 Gif/Yvette Cedex, France \\ email: clement.baruteau@cea.fr \\ ${ }^{2}$ IA-UNAM, Ciudad Universitaria, Apartado Postal 70-264, Mexico D.F. 04510, Mexico \\ email: fmasset@cea.fr
}

\begin{abstract}
We investigate the impact of the disk self-gravity on type I migration. We first show that considering a planet migrating in a disk without self-gravity can lead to a significant overestimate of the migration rate. Unbiased drift rates can be obtained only if the planet and the disk feel the same gravitational potential. We then confirm that the disk gravity slightly accelerates type I migration.
\end{abstract}

Keywords. accretion, accretion disks, gravitation, hydrodynamics, methods: numerical

\section{Introduction}

A low-mass planet embedded in a protoplanetary disk should undergo an orbital decay towards the central object. This process, known as type I migration, has been extensively studied analytically and numerically. Recent works on planet-disk interactions have challenged to include more physical ingredients and to yield precise estimates of the migration rate. Two of them investigated the impact of the disk self-gravity, but reported a priori contradictory statements: the disk self-gravity slows down migration for Nelson \& Benz (2003), whereas the disk gravity accelerates it for Pierens \& Huré (2005). This communication aims at clarifying and quantifying the impact of the disk gravity on type I migration.

\section{Torque estimate in a non self-gravitating disk}

Numerical setup. We performed two-dimensional calculations of a low-mass planet embedded in a non self-gravitating gaseous disk. The planet to primary mass ratio is $q=5 \times 10^{-6}$, and the softening length of the planet potential is $\varepsilon=0.3 h r_{p}$, where $h$ is the disk aspect ratio, taken uniform, and $r_{p}$ denotes the planet's orbital radius. In its unperturbed state, the disk has axisymmetric temperature and surface density profiles which read, respectively, $T(r)=T_{p}\left(r / r_{p}\right)^{-1}$ and $\Sigma(r)=\Sigma_{p}\left(r / r_{p}\right)^{-3 / 2}$, where $T_{p}$ and $\Sigma_{p}$ denote the temperature and the surface density at $r=r_{p}$. Since $\Sigma$ decreases as $r^{-3 / 2}$, and the disk is described by an isothermal equation of state, the corotation torque cancels out (Masset 2001, Baruteau \& Masset 2008). The torque exerted by the disk on the planet therefore reduces to the differential Lindblad torque. The disk feels the gravity of the central star and of the planet, while two situations are considered for the planet:

- It is held on a fixed circular orbit. In this case (the fixed case), the planet only feels the star gravity. The planet and the disk therefore feel the same gravitational potential. This situation has been contemplated for analytical estimates of the migration rate (e.g. Tanaka et al. 2002). 
- It freely migrates in the disk. In this case (the free case), the planet feels the star and the disk gravity. Contrary to the fixed case, this situation is not self-consistent since the planet and the disk do not orbit under the same potential. Nevertheless, this situation is of interest since it corresponds to the standard scheme of all planet-disk simulations.

An unexpected result. We display in figure 1 the torque $\Gamma$ exerted on the planet when varying $\Sigma_{p}$ (we take $h=0.05, T_{p}$ is therefore fixed for this series). When the planet is on a fixed orbit, $\Gamma$ scales with $\Sigma_{p}$, as expected. However, the free case reveals two unexpected results: $\Gamma$ increases faster than linearly with $\Sigma_{p}$, and, for a given value of $\Sigma_{p}$, $\Gamma$ is far larger in the free case than in the fixed one.

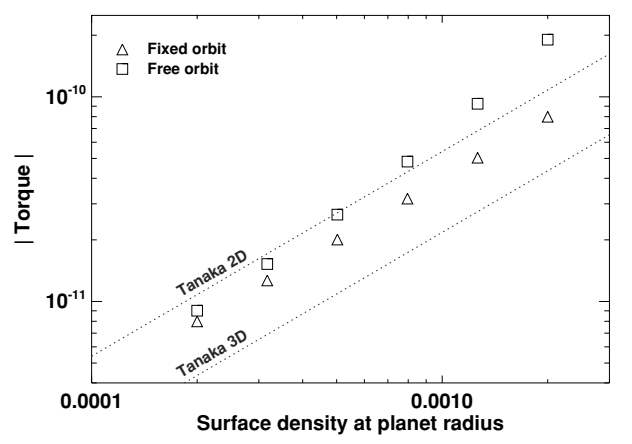

Figure 1. Torque exerted on a $M_{p}=5 \times 10^{-6} M_{*}$ planet mass by a non self-gravitating disk. The surface density at the planet radius is varied from one to ten times that of the MMSN. Triangles refer to the fixed case whereas squares refer to the free case (see text). The two dotted lines depict the two- and three-dimensional analytical estimates of Tanaka et al. (2002).

Shift of Lindblad resonances. These results can be explained with the relative positions of the Lindblad Resonances (LR) in the fixed and free cases. We display in figure 2a the locations $r_{\mathrm{ILR}}\left(r_{\mathrm{OLR}}\right)$ of an Inner (Outer) LR, when the planet is on a fixed orbit. They are given by $r_{\mathrm{ILR}}=\Omega^{-1}\left(\Omega_{\mathrm{ILR}}\right)$ and $r_{\mathrm{OLR}}=\Omega^{-1}\left(\Omega_{\mathrm{OLR}}\right)$, with $\Omega(r)$ the disk's rotation profile (solid curve), and with $\Omega_{\mathrm{ILR}}\left(\Omega_{\mathrm{OLR}}\right)$ the frequency of the ILR (OLR), simply deduced from the planet frequency $\Omega_{p}$. When the planet is on a free orbit (figure $2 \mathrm{~b}$ ), its frequency is larger than in the fixed case, depending on the mass distribution of the disk (Baruteau \& Masset 2007). Thus, the frequencies of the LR are also larger in the free case, and all the resonances are shifted inwards.

Torque discrepancy. The free situation induces a spurious inward shift of all the LR, which reduces the (positive) inner torque, and increases the (negative) outer one. Thus, the (negative) total torque is artificially larger in the free case. This torque overestimate increases with $\Sigma_{p}$ and can typically reach a factor of two (figure 1). In order to give unbiased torque estimates, the planet and the disk must feel the same potential.

\section{Torque estimate in a self-gravitating disk}

Impact of the disk gravity. In addition to the star gravity, the disk gravity is now felt by both the planet and the disk. The impact of the disk gravity is three-fold:

(a) It increases the planet frequency, as already stated in section 2 . This shifts all the LR inwards (figure $2 b$ ).

(b) Similarly, it increases the disk frequency, which shifts all the LR outwards (see figure 3). The increase of the planet and of the disk frequencies induces two shifts that 

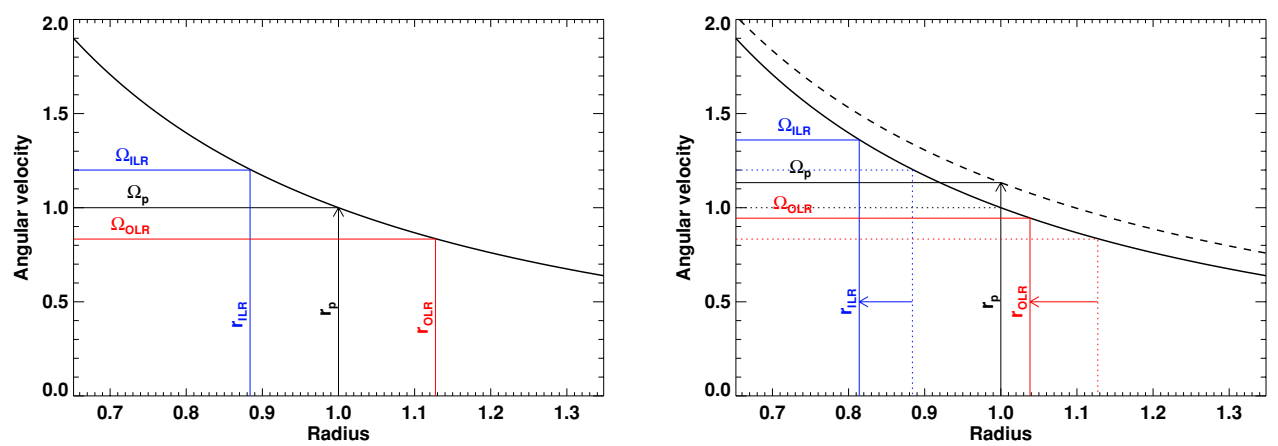

Figure 2. Location of two Lindblad resonances in the fixed case (left panel) and in the free case (right panel): the ILR of $m=6\left(\Omega_{\mathrm{ILR}}=6 / 5 \Omega_{p}\right)$, and the OLR of $m=5\left(\Omega_{\mathrm{OLR}}=5 / 6 \Omega_{p}\right)$. The disk's rotation profile is depicted without self-gravity (solid curve) and with self-gravity (dashed curve, right panel). The difference between both profiles has been exaggerated to improve legibility. In the right panel, arrows highlight the shift of the LR with respect to the left panel.

nearly compensate, and the net impact on the torque is negligible (Baruteau \& Masset 2007).

(c) It yields an additional term in the dispersion relation of density waves, which shifts the LR towards the planet location. This shift prevails over the sum of the two previous shifts (Pierens \& Huré 2005), and the disk gravity should enhance the torque.

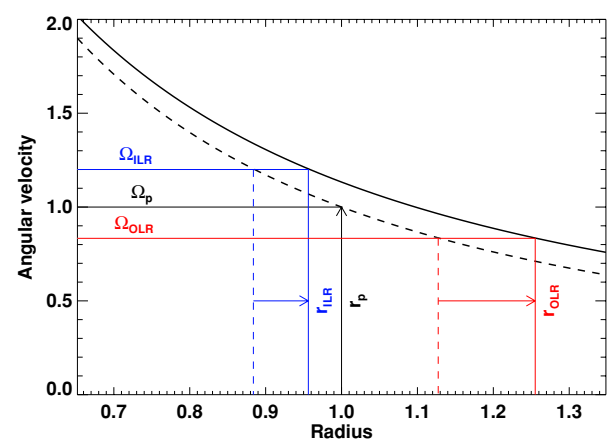

Figure 3. Same as figure 2, except that we examine the shift of the LR when the disk is self-gravitating (its rotation profile is now the solid, upper curve). The frequency of the planet, and therefore that of the LR, is the same as in figure $2 \mathrm{a}$.

Solving the contradiction. The torques obtained with self-gravitating calculations are displayed in figure $4 \mathrm{a}$. The results of figure 1 are overplotted for comparison:

- Comparing with the results of the self-consistent fixed case, as in Pierens \& Huré (2005), confirms that the disk gravity slightly accelerates type I migration.

- Comparing with the results of the biased free case, as done by Nelson \& Benz (2003), would also lead us to conclude that the disk self-gravity slows down migration significantly. However, this conclusion is misleading since it compares a self-consistent situation to a biased one.

What if one discards the disk gravity? The torque increase due to the disk gravity can be exclusively accounted for by a shift of the LR, and, under some limitations, be reproduced with an anisotropic pressure tensor, without self-gravity (Baruteau \& Masset 

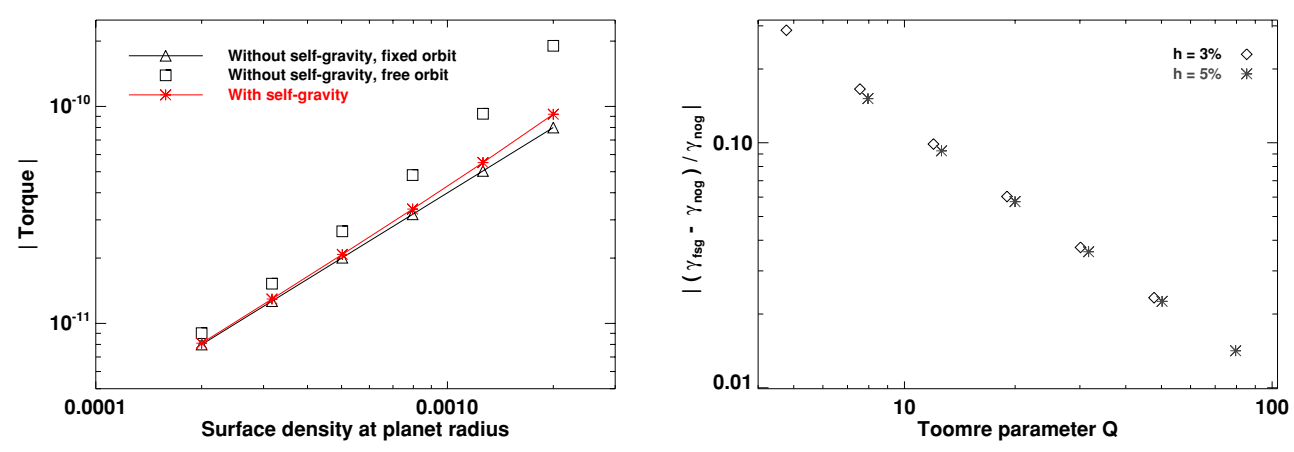

Figure 4. Left: same as figure 1, except that the results of the self-gravitating calculations are overplotted (stars). Right: relative difference of the torques obtained with the self-gravitating calculations $\left(\gamma_{\mathrm{fsg}}\right)$ and the calculations without disk gravity $\left(\gamma_{\mathrm{nog}}\right.$, those of the fixed case $)$, versus the Toomre parameter at the planet location.

2007). For a given softening length, the relative difference of the torques without and with disk gravity depends only on the Toomre parameter $Q$ at the planet location. It does not depend on the global mass distribution of the disk. Figure $4 \mathrm{~b}$ shows that this relative difference scales with $Q^{-1}$, at least for high to moderate values of $Q$. This figure yields a quantitative estimate of the torque underestimate if one discards the disk gravity (here for $\varepsilon=0.3 h r_{p}$, see Baruteau \& Masset (2007) for a study of its dependence with the softening length).

\section{Acknowledgements}

C.B. thanks Andrés González Carmona, Takayuki Muto and Hui Zhang for fruitful discussions at the symposium.

\section{References}

Baruteau, C. \& Masset, F. 2007, submitted to ApJ

Baruteau, C. \& Masset, F. 2008, ApJ, 672, 1054

Masset, F. S. 2001, ApJ, 558, 453

Nelson, A. F. \& Benz, W. 2003, ApJ, 589, 578

Pierens, A. \& Huré, J.-M. 2005, A $\& A$, 433, L37

Tanaka, H., Takeuchi, T., \& Ward, W. R. 2002, ApJ, 565, 1257 